\title{
Silencing of FABP3 leads to apoptosis-induced mitochondrial dysfunction and stimulates Wnt signaling in zebrafish
}

\author{
YAO-QIU LIU ${ }^{1 *}$, GUI-XIAN SONG ${ }^{2 *}$, HAI-LANG LIU ${ }^{2}$, XUE-JIE WANG $^{3}$, YA-HUI SHEN ${ }^{1}$, \\ LI-JUAN ZHOU ${ }^{2}$, JIN JIN $^{2}$, MING LIU ${ }^{2}$, CHUN-MEI SHI ${ }^{1}$ and LING-MEI QIAN ${ }^{2}$ \\ ${ }^{1}$ State Key Laboratory of Reproductive Medicine, Department of Pediatrics, Nanjing Maternity and Child Health Hospital \\ Affiliated to Nanjing Medical University, Nanjing, Jiangsu 210029; ${ }^{2}$ Department of Cardiology, \\ The First Affiliated Hospital of Nanjing Medical University, Nanjing, Jiangsu 210029; \\ ${ }^{3}$ Department of Emergency, Subei People Hospital, Yangzhou, Jiangsu 225001, P.R China
}

Received March 11, 2013; Accepted July 4, 2013

DOI: $10.3892 / \mathrm{mmr} .2013 .1586$

\begin{abstract}
Fatty acid binding protein 3 (FABP3, also termed heart-type fatty acid binding protein) is a member of the intracellular lipid-binding protein family that may be essential in fatty acid transport, cell growth, cellular signaling and gene transcription. Previously, we demonstrated that FABP3 was involved in apoptosis-associated congenital cardiac malformations; however, its mechanism of regulation remains unclear. Apoptosis has increasingly been considered to be important in cardiac development. In the present study, a zebrafish model was used to investigate the involvement of FABP3-morpholino (MO)-induced apoptosis and mitochondrial dysfunction in cardiac development. During the early stages of cardiac development, injection of FABP3-MO into zebrafish resulted in significant impairment in cardiac development and promoted the rate of apoptosis which was correlated with significant dysfunction of the mitochondria. For example, the ATP content was markedly decreased at 24 and $48 \mathrm{~h}$ post-fertilization (pf), reactive oxygen species production was significantly enhanced at 24 and $48 \mathrm{~h}$ pf and the mitochondrial DNA copy number was reduced at 24, 48 and $72 \mathrm{~h}$ pf. Additionally, Nkx2.5 expression was upregulated in FABP3-MO zebrafish, and Wnt signaling molecules (Wnt1, Wnt5 and Wnt11) were also
\end{abstract}

Correspondence to: Dr Ling-Mei Qian, Department of Cardiology, The First Affiliated Hospital of Nanjing Medical University, 140 Hanzhong Road, Nanjing, Jiangsu 210029, P.R. China E-mail: 1mqian@njmu.edu.cn

Dr Chun-Mei Shi, Department of Cardiology, Nanjing Maternity and Child Health Hospital Affiliated to Nanjing Medical University, 300 Guangzhou Road, Nanjing, Jiangsu 210029, P.R. China

E-mail: shichunmei_87@163.com

${ }^{*}$ Contributed equally

Key words: mitochondrial dysfunction, fatty acid-binding protein 3, zebrafish, cardiac development, apoptosis, Wnt signaling highly expressed in FABP3-MO zebrafish at 24, 48 and $72 \mathrm{~h}$ pf. In conclusion, the results indicated that FABP3 knockdown exhibited significant toxic effects on cardiac development and mitochondrial function, which may be responsible for the knockdown of FABP3-induced apoptosis. Apoptosis was one of the mechanisms underlying this effect, and was correlated with the activation of Wnt signaling. These studies identified FABP3 as a candidate gene underlying the etiology of congenital heart defects.

\section{Introduction}

The vertebrate multichambered heart is the first organ to form during development (1). The zebrafish offers several distinct advantages as a genetic and embryonic model system to study cardiovascular disease. Zebrafish mutations have revealed specific genetic requirements for cardiac fate determination, migration, fusion, tube assembly, looping and remodeling. These processes maintain functional integrity and cardiac function may affect aspects of cardiac morphogenesis (2). In particular, the spatial and temporal coordination is essential for the heart to ensure proper cardiac function throughout development. Therefore, the investigation of the genetic regulation of cardiogenesis is popular among developmental biologists using a variety of model organisms from flies to zebrafish.

Fatty acid binding protein 3 (FABP3), also termed heart-type fatty acid binding protein, may be essential in fatty acid transport, cell growth, cellular signaling and gene transcription $(3,4)$. FABP3 is upregulated during the terminal differentiation of mouse cardiomyocytes (5). In our previous studies, FABP3 was differentially expressed between ventricular septum defect myocardium and normal ventricular septum myocardium (6). In addition, the inhibition of FABP3 expression promoted cell apoptosis and led to mitochondrial dysfunction in P19 cells (embryonic murine carcinoma cells) (7). However, the precise role of FABP3 in cardiac development remains to be elucidated.

Cardiac development is controlled by evolutionarily conserved transcription factors which connect signaling pathways with genes for muscle growth, patterning and contractility, which involves a series of molecular and 
morphogenetic events. Cardiac lineage commitment occurs between the first and second stages of development depending on the expression of cardiac transcription factors, such as Nkx2.5, GATA4, MEF2C and dHAND, in which Nkx2.5 is involved in embryo heart development and stem cell cardiomyogenesis (8). The Wnt receptor signaling pathway is also known to be involved in every aspect of embryonic development, including cell-fate specification, proliferation, survival, migration and adhesion, particularly in cardiac development and differentiation $(9,10)$.

Furthermore, apoptosis is an essential event in vertebrate organ formation and the development process. Apoptosis participates in the fundamental aspects of morphology development, such as endocardia, epicardia and neural crest formation, and is also essential in embryonic development and adult tissue homeostasis $(11,12)$. In addition, gain and loss of function studies demonstrated that apoptosis may result in congenital heart defects $(12,13)$. It has been determined that mitochondria are involved in apoptosis by inducing a variety of pro-apoptotic conditions, including the release of caspase activators, altered cellular oxidation-reduction and participation of pro- and anti-apoptotic Bcl-2 family proteins (14).

In the present study, zebrafish were used as a model to investigate the involvement of FABP3 in cardiac development and to determine the effects of FABP3 knockdown on cell apoptosis and mitochondrion function in vivo. In addition, the gene expression profile of the Wnt signaling pathway and Nkx2.5 were analyzed to determine whether FABP3 knockdown exhibits an effect on this pathway in zebrafish cardiac development.

\section{Materials and methods}

Embryo maintenance and transplantation. Wild type (WT) zebrafish stocks were obtained from the Model Animal Research Center of Nanjing University (Nanjing, Jiangsu, China). Embryos were obtained from natural spawning of wild type adults and were grown at $28.5^{\circ} \mathrm{C}$ in embryo medium which was changed twice per day, as previously described (15). Morphological features were used to determine the embryonic developmental stage, as described by Kimmel et al (16). Embryos older than $24 \mathrm{~h}$ post-fertilization (pf) were incubated in $0.003 \%$ phenylthiourea to inhibit pigment formation.

Morpholinos (MOs) and microinjection. MOs were purchased from Gene Tools (Philomath, OR, USA). The FABP3-MO was designed against the ATG start site of FABP3 to block its translation (FABP3-MO; 5'-ACG TGC CGA TAA AAG CGT CTG CCA T-3'). A standard control MO (Wt-MO; 5'-CCT CTT ACC TCA GTT ACA ATT TAT A-3') served as a negative control (17). MO oligomers were dissolved in $0.3 \mathrm{X}$ $2 \mathrm{mmol} / 1$ Danieaus's solution [58 mmol/1 NaCl, $0.7 \mathrm{mmol} / 1$ $\mathrm{KCl}, 0.4 \mathrm{mmol} / 1 \mathrm{MgSO}_{4}, 0.6 \mathrm{mmol} / 1 \mathrm{Ca}\left(\mathrm{NO}_{3}\right)_{2}, 5.0 \mathrm{mmol} / \mathrm{l}$ $\mathrm{N}$-(2-hydroxyethyl) piperazine-N'-(2-ethanesulfonic acid) (HEPES), pH 7.6] (18). The oligomers were diluted to their final concentration in $0.2 \mathrm{~mol} / \mathrm{l} \mathrm{KCl}$ and $0.5 \%(\mathrm{w} / \mathrm{v})$ phenol red (Sigma-Aldrich, St. Louis, MO, USA). Injection of $5.0 \mathrm{ng}$ MO into single to four-cell stage zebrafish embryos using back-filled fine borosilicate glass capillary needles (Corning Inc., Corning, NY, USA) was performed as previously described (18). Following MO injection, embryos were incubated at $28.5^{\circ} \mathrm{C}$ for up to 3 days pf.

Measurements of heart development. Following microinjection of MOs into zebrafish embryos, the embryos were incubated at $28.5^{\circ} \mathrm{C}$ for up to 4 days pf. In order to determine the effect of time on FABP3-MO-related morphological abnormalities of the heart, histological sections were used to identify heart-specific phenotypes. Larvae were anesthetized with $0.05 \%$ tricaine (3-aminobenzoic acid ethylester) and fixed in $4 \%$ paraformaldehyde in $0.1 \mathrm{M}$ Sorenson's buffer (pH 7.4). Larvae were washed in $0.01 \mathrm{M}$ phosphate-buffered saline (PBS; pH 7.2), dehydrated in a graded series of ethanol, infiltrated and embedded in blocks. Each block was serially transverse-sectioned at $5 \mu \mathrm{m}$. Sections were hydrated, stained with Harris' hematoxylin and eosin Y, dried and mounted in neutral balsam. For larvae within the block, measurements were made for the heart when the central portion of the heart was visible. In each group, six larvae were assessed for heart layer thickness and cell density at $96 \mathrm{~h} \mathrm{pf} \mathrm{(4} \mathrm{days} \mathrm{pf).} \mathrm{All} \mathrm{other}$ slides of the selected heart were imaged at a final magnification of x1000 with a Pixera Pro 600ES digital camera (Los Gatos, CA, USA) attached to a JD-801 microscope (Olympus, Tokyo, Japan).

Apoptosis assay. Following injection of $5.0 \mathrm{ng} \mathrm{MO}$ into single to four-cell stage zebrafish embryos, zebrafish were harvested using collagenase I (Sigma-Aldrich) at 24 and $48 \mathrm{~h}$ pf, and washed with PBS. The single-cell suspensions of zebrafish were centrifuged, resuspended in $1 \mathrm{ml}$ binding buffer and stained with $10 \mu \mathrm{l}$ Annexin V-fluorescein isothiocyanate (FITC; BioVision, Mountain View, CA, USA) and $10 \mu$ propidium iodide at room temperature for $15 \mathrm{~min}$. The stained cells were analyzed immediately using flow cytometry as described previously (19).

ATP production assay. Zebrafish larvae were digested with collagenase I (Sigma-Aldrich) to obtain single-cell suspensions. The ATP content of the zebrafish was measured using a luciferase-based luminescence assay kit (Biyuntian, Nantong, Jiangsu, China). Briefly, the single-cell suspensions of zebrafish were homogenized in ice-cold ATP-releasing buffer and light emission was recorded for $30 \mathrm{sec}$ using a photon-counting luminometer (Turner Biosystems, Sunnyvale, CA, USA) pf at 24 and $48 \mathrm{~h}$ pf. The relative ATP level was normalized to the protein concentration of each sample, as determined by a bicinchoninic acid assay.

Reactive oxygen species assay. Intracellular reactive oxygen species (ROS) generation was determined using 6-carboxy2,7-dichlorodihydrofluorescein diacetate $\left(\mathrm{H}_{2}\right.$-DCFDA; Sigma-Aldrich), as previously described (20). Briefly, following injection with 5.0 ng MO into single to four-cell stage zebrafish embryos, the embryos were collected pf at 24 and $48 \mathrm{~h}$ pf. The digested single-cell suspensions of zebrafish were washed and incubated with $\mathrm{H}_{2}$-DCFDA for $20 \mathrm{~min}$. Subsequent to this, the suspensions were washed several times and harvested in PBS. The fluorescence of $\mathrm{H}_{2}$-DCFDA was detected using a fluorescence-activated cell sorter (FACS; excitation, $488 \mathrm{~nm}$; emission, $530 \mathrm{~nm})$. 
qPCR for the assessment of mitochondrial DNA (mtDNA) concentration. The relative quantities of mtDNA were determined by qPCR, which was performed using an Applied Biosystems 7500 Sequence Detection system (ABI 7500 SDS; Applied Biosystems, Foster City, CA, USA) according to the manufacturer's instructions (21). In brief, DNA was isolated from the zebrafish following microinjection $24,48,72 \mathrm{~h}$ pf using the Wizard ${ }^{\circledR}$ Genomic DNA purification kit DNA extraction kit (Promega, Madison, WI, USA) and quantified using a NanoDrop 2.0 spectrophotometer (Thermo Scientific, Foster City, CA, USA). Two primer sets (listed in Table I) were used for PCR analysis. A 125 bp mtDNA fragment within the cytochrome $\mathrm{b}(C Y T B)$ gene was used for the quantification of mtDNA. We previously cloned the PCR product into the plasmid pMD18-T and verified it by DNA sequencing. Plasmid standards of known copy number were used to generate a log-linear standard curve, from which the CYTB copy number was determined in the samples by qPCR. The results were normalized to a $168 \mathrm{bp}$ region of the nuclear gene for 28S rRNA using a standard curve generated from a plasmid containing the $28 \mathrm{~S}$ fragment. The ratio of mtDNA to nuclear DNA was determined to reflect the concentration of mitochondrial DNA per cell.

qPCR for the assessment of Nkx2.5 and molecules of the Wnt signalling pathway. Total RNA was extracted from zebrafish using TRIzol reagent (Invitrogen Life Technologies, Carlsbad, CA, USA), and the extracted RNA was quantified using a NanoDrop 2.0 spectrophotometer (Thermo Scientific). Complimentary DNA was synthesized from $2 \mu \mathrm{g}$ total RNA by using an AMV Reverse Transcriptase kit (Promega A3500; Promega, Madison, WI, USA) according to the manufacturer's instructions. qPCR was performed using SYBR-Green PCR Master mix (Applied Biosystems) on an Applied Biosystems 7500 Sequence Detection System (ABI 7500 SDS). qPCR reaction conditions were $95^{\circ} \mathrm{C}$ for $10 \mathrm{~min}$ followed by 40 cycles at $94^{\circ} \mathrm{C}$ for $20 \mathrm{sec}, 60^{\circ} \mathrm{C}$ for $20 \mathrm{sec}$ and $72^{\circ} \mathrm{C}$ for $30 \mathrm{sec}$ with a final extension of $7 \mathrm{~min}$ at $72^{\circ} \mathrm{C}$. NK2 homeobox $5(\mathrm{Nkx} 2.5)$, Wnt1, Wnt5a and Wnt11 expression was normalized against $\beta$-actin using the comparative CT method to determine the relative changes in mRNA expression in the target sample. The sequences of the primers used for each gene are shown in Table I.

Statistical analysis. All data are presented as the mean \pm standard deviation of three independent experiments. Statistical significance was assessed using the independent-samples t-test using the statistical software package SPSS 16.0 (SPSS, Inc., Chicago, IL, USA). $\mathrm{P}<0.05$ was considered to indicate a statistically significant difference.

\section{Results}

FABP3-MO impairs heart development. To investigate the function of FABP3 in the establishment of cardiac development, a loss-of-function study was performed using a previously characterized anti-FABP3 (ATG) MO (in our previous original study: knockdown of FABP3 impaired cardiac development in zebrafish through the retinoic acid signaling pathway). The MO oligonucleotides were injected into single to four-cell stage zebrafish embryos directly and is effective up to 3-4 days pf (18). Thus, in the present study, embryos were injected with $0.5 \mathrm{ng}$ MO targeting FABP3 translation. Cardiac development is complete at $\sim 96 \mathrm{~h} \mathrm{pf}$ (4 days pf) (22), and the thickness of the heart is normally $\sim 30-40 \mu \mathrm{m}$ at 4 days pf. By 4 days pf, we observed defective phenotypes of embryos resulting from FABP3-MO by using a series of continuous section slides by tissue cutting (thickness, $\sim 5 \mu \mathrm{m})$. Compared with the Wt-MO group, transverse sections of the heart of zebrafish larvae at 4 days pf displayed defective phenotypes, including a thin atrial-ventricular wall, a change in the relative position of the atrium and ventricle, an expansion of the diameter of the atrioventricular cavity and an alteration in the morphology of the bulbus arteriosus (Fig. 1). In addition to these defects, pericardial edema, developmental delay and incomplete looping of the developing heart were also observed in FABP-MO zebrafish as we demonstrated previously (23).

FABP3-MO promotes zebrafish cell apoptosis. To study the effect of FABP3-MO on zebrafish cell apoptosis, the zebrafish larvae were harvested at 24 and $48 \mathrm{~h}$ pf using collagenase I. The single-cell suspensions of zebrafish larvae were quantified by flow cytometric analysis subsequent to staining with Annexin V-FITC. The results demonstrated that FABP3-MO promoted zebrafish cell apoptosis compared with Wt-MO (Fig. 2; ${ }^{*} \mathrm{P}<0.05$ ).

Effects of FABP-MO on cellular ATP production in zebrafish. To further investigate whether FABP3 knockdown in zebrafish leads to impaired mitochondrion function, the ATP content was assayed in the FABP3-MO and Wt-MO groups in zebrafish at 24 and $48 \mathrm{~h}$ pf. As shown in Fig. 3 , the zebrafish ATP content in the FABP3-MO group was significantly decreased compared with the Wt-MO group at 24 and $48 \mathrm{~h} \mathrm{pf}($ ("P<0.05). The results showed that FABP3 knockdown in zebrafish decreased the total cellular ATP production.

FABP3-MO affects zebrafish intracellular ROS levels. ROS, a by-product of the electron transport chain, is predominantly produced by mitochondria (24). As shown in Fig. 4, in contrast with Wt-MO injected zebrafish, embryos injected with FABP3-MO at 24 and $48 \mathrm{~h}$ pf induced a significantly increased concentration of intracellular ROS levels, as indicated by increased fluorescence in the presence of the compound DCFDA $\left(2^{\prime}, 7^{\prime}\right.$-dichlorofluorescein diacetate; $\left.{ }^{\prime} \mathrm{P}<0.05\right)$. This result further demonstrated that FABP3-MO impaired mitochondrion function in zebrafish.

Effects of FABP3-MO on mtDNA copy number in zebrafish. In addition to the ATP content and ROS production detection of FABP3-MO injected zebrafish, the mitochondrial DNA copy number is regarded to indicate the cellular mitochondrion number and may be a possible marker of mitochondrion function. We measured mitochondrial and genomic DNA in FABP3-MO and Wt-MO injected embryos using qPCR. The results demonstrated that the mtDNA copy number was decreased in the FABP3-MO group compared with the Wt-MO group at three time points, in particular, the mtDNA copy number was significantly decreased at $24 \mathrm{~h}$ pf in the 
Table I. Sequences for primer sets used in qPCR gene expression analysis.

\begin{tabular}{lll}
\hline Gene name & \multicolumn{1}{c}{ Sense primer $\left(5^{\prime}-3^{\prime}\right)$} & \multicolumn{1}{c}{ Antisense primer $\left(5^{\prime}-3^{\prime}\right)$} \\
\hline CYTB & TATTCGCATACGCCATTC & TGCTATTCCTCGCTGTTT \\
$28 \mathrm{~s}$ & ATATGCGTCCGTCCCACTT & ATCGCCACCTTGACTTCG \\
Wnt 1 & GCTGAGAGGGAGGTGACAA & TACAAAGGCAGGAGGGAATG \\
Wnt5a & TACGCCTTCTTCAAGCATCC & CTCTTTGCGCTTTTCTGTCC \\
Wnt 11 & GTAAGTGCCATGGGGTGTCT & GCTTCCAAGTGAAGGCAAAG \\
Nkx 2.5 & GCTTTTACGCGAAGAACTTCC & GATCTTCACCTGTGTGGAGG \\
$\beta$-actin & CAACAGAGAGAAGATGACACAGATCA & GTCACACCATCACCAGAGTCCATCAC
\end{tabular}

A

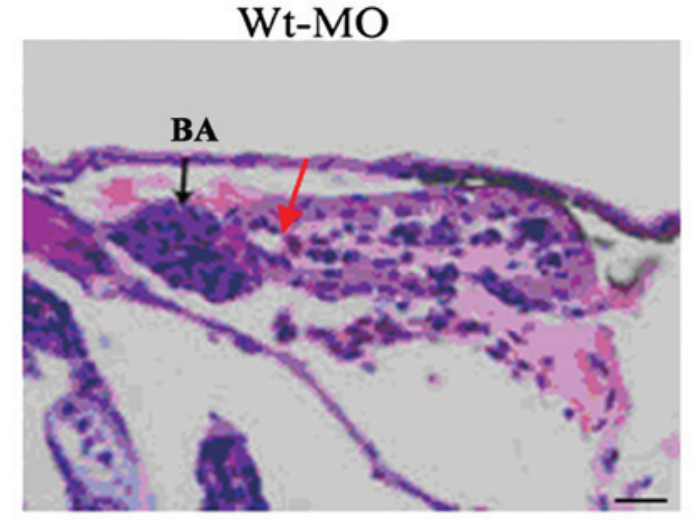

BA:bulbus arteriosus
B

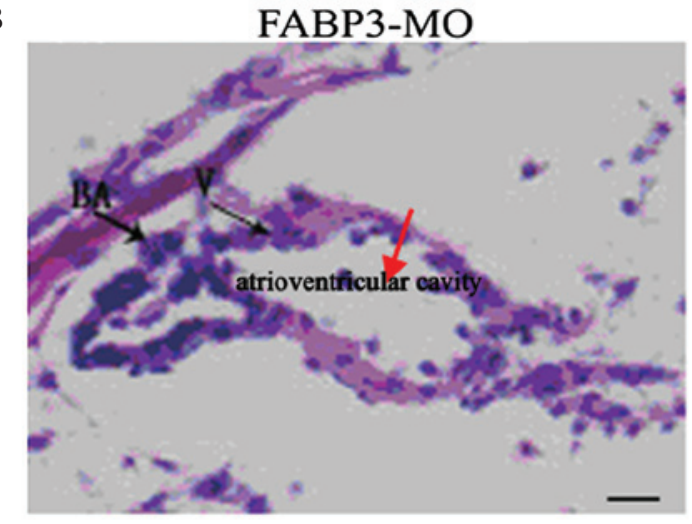

V:ventricle

Figure 1. Histological sections of developed heart 4 days pf. (A and B) Wt-MO and FABP3-MO are represented respectively. Compared with the Wt-MO group, embryos injected with $0.5 \mathrm{ng}$ FABP3-MO following 4 days pf displayed defective phenotypes, including a thin atrial-ventricular wall, a change in the relative position of the atrium and $\mathrm{V}$, an expansion of the diameter of the atrioventricular cavity and an alteration in the morphology of the BA. Scale bar, $20 \mu \mathrm{m}$. BA, bulbus arteriosus; V, ventricle; pf, post-fertilization; Wt-MO, wild type-morpholinos; FABP3-MO, fatty acid binding protein 3.

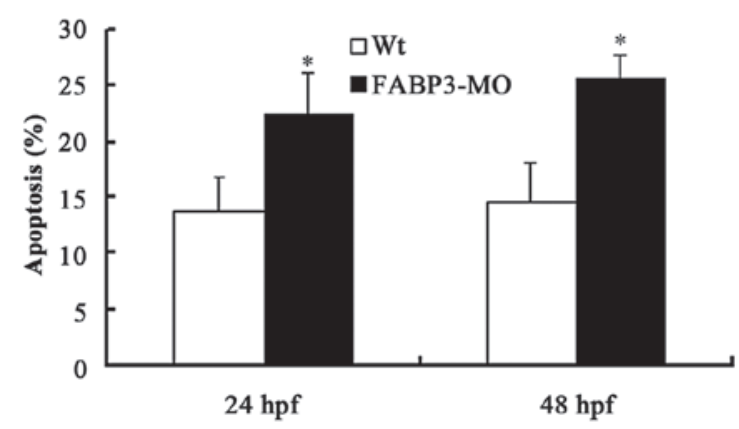

Figure 2. Effect of FABP3 knockdown on zebrafish apoptosis. Zebrafish larvae were harvested at 24 and $48 \mathrm{~h}$ pf using collagenase I. Apoptosis was assayed by an Annexin V-fluorescein isothiocyanate assay. Suppression of FABP3 promoted apoptosis compared with that in the Wt-MO group at 24 and $48 \mathrm{~h}$ pf. The results represent the mean $\pm \mathrm{SD}$ of three independent experiments $(\mathrm{n}=60)$. ${ }^{*} \mathrm{P}<0.05$. FABP3, fatty acid binding protein 3 ; pf, postfertilization; MO, morpholinos; WT, wild-type.

FABP-3MO group (Fig. 5; "P<0.05). However, there was no significant difference in mtDNA copy number at 48 and $72 \mathrm{~h}$ pf between the two groups (Fig. 5; P>0.05).

Alteration of expression patterns of Nkx2.5 and Wnt signaling molecules in FABP-MO zebrafish. To further investigate the effect of FABP3-MO on cardiac development, the expres-

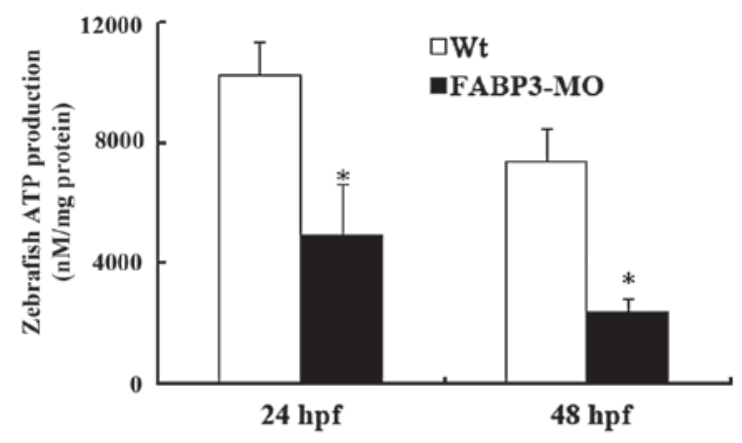

Figure 3. Effect of FABP-MO on cellular ATP production in zebrafish. Cellular ATP production was measured by a luciferase-based luminescence assay and was normalized to protein concentration in zebrafish larvae digested with collagenase I. ${ }^{*} \mathrm{P}<0.05$ compared with that in the Wt-MO group at 24 and $48 \mathrm{~h} \mathrm{pf}(\mathrm{n}=60)$. These data were based on four independent experiments. FABP3, fatty acid binding protein 3 ; pf, post-fertilization; MO, morpholinos; WT, wild-type.

sion level of the cardiac development-specific gene, $\mathrm{Nkx} 2.5$ (the earliest known marker of vertebrate heart development), was determined (25). Notably, as shown in Fig. 6A, qPCR results demonstrated that $\mathrm{Nkx} 2.5$ was upregulated in zebrafish embryos injected with FABP3-MO compared with those injected with Wt-MO. 


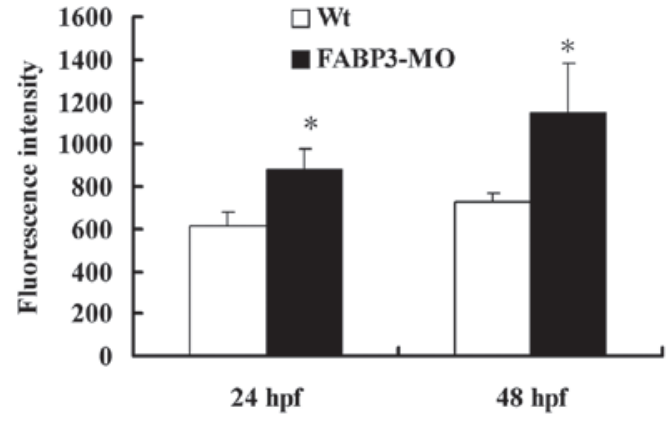

Figure 4. FABP3-MO affected zebrafish intracellular ROS levels. ROS levels were analyzed by measurement of the fluorescence of DCFDA using a fluorescence activated cell sorter. Embryos injected with FABP3-MO at 24 and $48 \mathrm{~h}$ pf induced a high concentration of intracellular ROS levels compared with those injected with Wt-MO $(\mathrm{n}=60)$. ${ }^{*} \mathrm{P}<0.05$. FABP3, fatty acid binding protein 3; pf, post-fertilization; MO, morpholinos; WT, wild-type; ROS, reactive oxygen species; DCFDA, 2',7'-dichlorofluorescein diacetate.

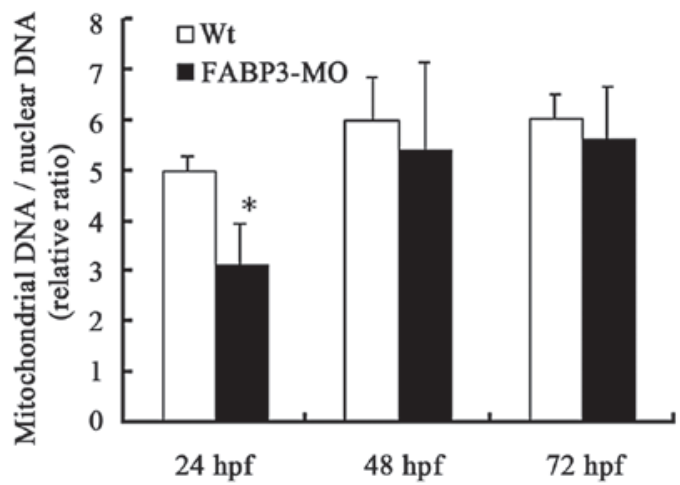

Figure 5. Effect of FABP3-MO on mtDNA in zebrafish. Total DNA was extracted at 24,48 and $72 \mathrm{~h} \mathrm{pf}$, and mtDNA content was determined by qPCR analysis with primers designed to target cytochrome $\mathrm{b}$ and $28 \mathrm{~S}$ rRNA genes $(\mathrm{n}=60)$. The mtDNA copy number was dramatically decreased at $24 \mathrm{~h} \mathrm{pf}$ in the FABP3-MO group, ${ }^{*} \mathrm{P}<0.05$. By 48 and $72 \mathrm{~h}$ pf, there was no significant difference between the two groups. FABP3, fatty acid binding protein 3; pf, post-fertilization; MO, morpholinos; mtDNA, mitochondrial DNA.

In addition, the mechanism through which FABP3 knockdown may affect cardiac development was investigated by focusing on the Wnt signaling pathway. Wnt signals exhibit developmental stage-specific and biphasic involvement in cardiomyogenesis and are also involved in heart disease (26-28). Wnt signaling consists of the canonical (Wnt1) and non-canonical (Wnt5 and Wnt11) Wnt signaling pathways. Thus, Wnt1, Wnt5a and Wnt11 were chosen to investigate the involvement of Wnt signaling in zebrafish cardiac development in injected FABP3-MO zebrafish embryos using qPCR at 24, 48 and $72 \mathrm{~h}$ pf, respectively. As shown in Fig. 6B-D, the expression pattern of the three Wnt signaling molecules was enhanced in FABP3-MO injected zebrafish at 24, 48 and $72 \mathrm{~h}$ pf. These results indicated that FABP3-MO affected the Wnt signaling pathway which may also have contributed to abnormal cardiac development.

\section{Discussion}

The heart is the first organ to form and function in vertebrate embryo development. The transparency of the zebrafish embryo
A

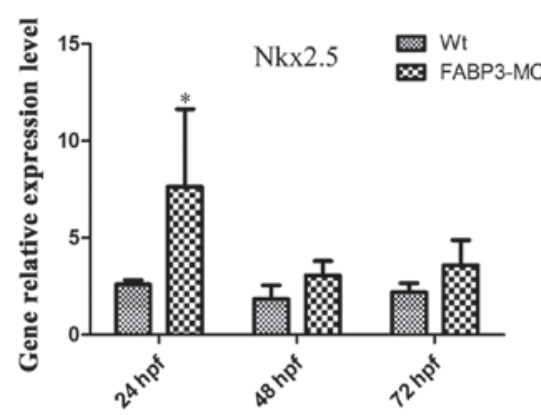

B

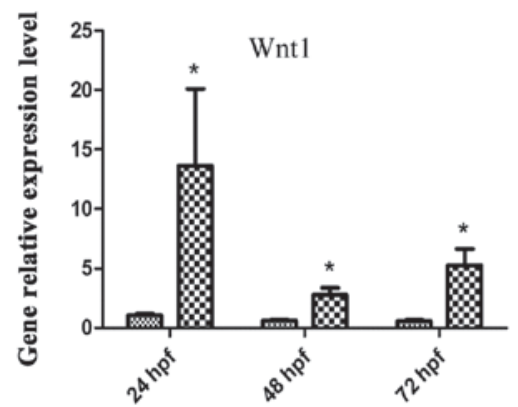

C

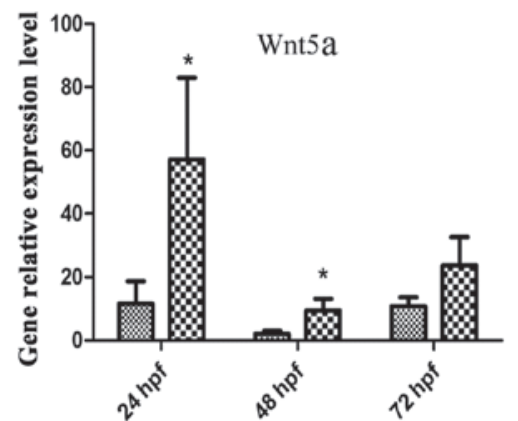

D

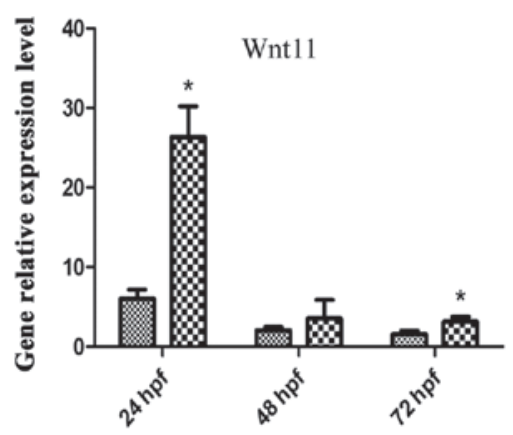

Figure 6. Gene expression profiles of Nkx2.5 and Wnt genes during cardiac development following injection of FABP3-MO zebrafish. RNA was harvested from FABP3-MO and Wt-MO injected zebrafish, respectively, at different time points, as indicated. Total mRNA was normalized with glyceraldehyde 3-phosphate dehydrogenase mRNA $(n=60)$. Gene expression profiles of (A) Nkx2.5 and (B-D) Wnt genes are shown. Values are presented as the mean \pm SD from three independent experiments. ${ }^{*} \mathrm{P}<0.05$ compared with WT. FABP3, fatty acid binding protein 3; MO, morpholinos; WT, wildtype; pf, post-fertilization.

and the fact that zebrafish survive without circulating blood until the larval stage, are advantages that allow the high-resolution visualization of the heart during its rapid development and facilitates the extended study of heart dysfunction (29-31). Therefore, zebrafish are commonly selected as model organisms in the study of cardiac development.

It has previously been demonstrated that FABP3 was highly expressed in patients with ventricular-septal defects, when 
compared with normal controls (6). It has also been demonstrated that inhibition or overexpression of FABP3 promoted cell apoptosis and resulted in mitochondrial dysfunction in P19 cells, which indicated that FABP3 promoted apoptosis through the induction of mitochondrial impairment in P19 cells (7). The function of FABP3 in cardiac development in vivo remains to be elucidated despite observations in vitro. In the present study, cardiac development was investigated in FABP3-MO injected zebrafish embryos and the cause of heart defects was studied at the molecular level.

Apoptosis is hypothesized to be important during the critical stages of heart development and excess apoptosis directly or indirectly results in congenital heart disease (CHD) (32). In the present study, results demonstrated that FABP3-MO promoted zebrafish cell apoptosis which was concordant with our previous study in vitro (23). Furthermore, over the past few decades, studies have focused on the correlation between mitochondrial function and apoptosis, including the release of caspase activators (such as cytochrome $c$ ), the alteration of cellular oxidation-reduction and the involvement of pro- and antiapoptotic Bcl-2 family proteins (14). Thus, in the present study, it was determined whether mitochondrial function was predominant in triggering or inhibiting apoptosis in zebrafish. In addition, FABP3 is predominantly involved in the mitochondrial energy metabolism and in transferring long-chain fatty acids to the mitochondria in the process of ATP production in myocardial cells (33). Therefore, ATP content, ROS production and mtDNA copy number was investigated in FABP3-MO zebrafish to determine the effect of FABP3-MO on mitochondrial function during the process of cardiac development. The results indicated that significant dysfunction in mitochondria was observed in FABP3-MO injected zebrafish, including a marked decrease in ATP content, enhanced ROS production and reduced mtDNA, which was concordant with the results of our in vitro experiments (23). In addition, the inhibition efficiency of the FABP3-MO was greatest at $48 \mathrm{~h}$ pf. Thus, mitochondrion dysfunction may be responsible for the development of FABP3-induced apoptosis in zebrafish.

In order to determine the mitochondrial function and apoptosis in FABP3-MO injected zebrafish, the effect of FABP3-MO on Nkx2.5 and Wnt gene expression was also determined. $\mathrm{Nkx} 2.5$ is a transcription factor which is essential for cardiac precursor formation and differentiation (34), regulates multiple aspects of cardiac cell structure, function, and development $(8,35)$. Deletion of this gene results in the failure of normal heart development and the disorder of cardiomyocyte maintenance (25). As the $\mathrm{Nkx} 2.5$ transcription factor is one of the earliest genes expressed in the heart cells (36), the expression level of $\mathrm{Nkx} 2.5$ was determined in FABP3-MO zebrafish. The results demonstrated that the expression level of $\mathrm{Nkx} 2.5$ was upregulated in FABP3-MO compared with Wt-MO injected zebrafish.

Furthermore, a large number of studies have demonstrated that blocking Wnt signaling (canonical and non-canonical) in the anterior mesoderm of Xenopus embryos influenced the expression of early cardiac genes, including Nkx2.5, Gata4 and Tbx5 $(37,38)$. Thus, to determine why the expression level of Nkx2.5 was enhanced, the expression profile of Wnt signaling molecule (Wnt1, Wnt5a and Wnt11) was analyzed. Concordant with the hypothesis, the expression pattern of the three Wnt signaling molecules were enhanced in FABP3-MO injected zebrafish. Moreover, Wnt signals exhibited developmental stage-specific and biphasic effects (both positive and negative) on cardiomyogenesis (26-28). At least ten types of FABPs have been identified in vertebrates (39), each FABPs gene showed a specific pattern of expression during development and in adulthood in mammals (40). Thus, FABP3-MO led to an abnormal expression profile in zebrafish. Additionally, Wnt/ $\beta$-catenin is pro-cardiogenic in the early precardiac mesoderm and inhibitory later in cardiac differentiation. The non-canonical Wnts are essential in cardiac morphogenesis. Recent studies from the Morrisey lab demonstrated that the non-canonical Wnts inhibit canonical Wnt signaling in heart development (41). Therefore, the balance of canonical and non-canonical Wnt was key in heart development. Dohn and Waxman (42) indicated that canonical Wnts promoted apoptosis through p53 and caspase-3 independent mechanisms in zebrafish, which suggested that FABP3-MO induced apoptosis in zebrafish, which may occur through the promotion of the Wnt signaling pathway.

In the present study, FABP3-MO led to defects in cardiac development of zebrafish embryos. In addition, the molecular mechanism underlying such defects involved in mitochondrial dysfunction induced-apoptosis. Moreover, these studies have demonstrated that Wnt activity is a key regulator of the morphogenesis of the heart.

\section{Acknowledgements}

This study was supported by grants from the National Natural Science Foundation of China (grant no. 81070138), the Natural Science Foundation of Jiangsu Province, China (grant no. BK2010582) and the Talent Foundation of Jiangsu Province, China (grant no. WSN-020).

\section{References}

1. Srivastava D: Making or breaking the heart: from lineage determination to morphogenesis. Cell 126: 1037-1048, 2006.

2. Glickman NS and Yelon D: Cardiac development in zebrafish: coordination of form and function. Semin Cell Dev Biol 13: 507-513, 2002.

3. Qian Q, Kuo L, Yu YT and Rottman JN: A concise promoter region of the heart fatty acid-binding protein gene dictates tissue-appropriate expression. Circ Res 84: 276-289, 1999.

4. Besnard P, Niot I, Poirier H, Clément L and Bernard A: New insights into the fatty acid-binding protein (FABP) family in the small intestine. Mol Cell Biochem 239: 139-147, 2002.

5. Tang MK, Kindler PM, Cai DQ, Chow PH, Li M and Lee KK: Heart-type fatty acid binding proteins are upregulated during terminal differentiation of mouse cardiomyocytes, as revealed by proteomic analysis. Cell Tissue Res 316: 339-347, 2004.

6. Zhang H, Zhou L, Yang R, et al: Identification of differentially expressed genes in human heart with ventricular septal defect using suppression subtractive hybridization. Biochem Biophys Res Commun 342: 135-144, 2006.

7. Shen YH, Song GX, Liu YQ, et al: Silencing of FABP3 promotes apoptosis and induces mitochondrion impairment in embryonic carcinoma cells. J Bioenerg Biomembr 44: 317-323, 2012.

8. Bruneau BG: Transcriptional regulation of vertebrate cardiac morphogenesis. Circ Res 90: 509-519, 2002.

9. Gessert S and Kühl M: The multiple phases and faces of wnt signaling during cardiac differentiation and development. Circ Res 107: 186-199, 2010.

10. Nusse R: Wnt signaling in disease and in development. Cell Res 15: 28-32, 2005. 
11. Thompson CB: Apoptosis in the pathogenesis and treatment of disease. Science 267: 1456-1462, 1995.

12. Poelmann RE and Gittenberger-de Groot AC: Apoptosis as an instrument in cardiovascular development. Birth Defects Res C Embryo Today 75: 305-313, 2005.

13. Fiorina P, Corradi D, Pinelli S, et al: Apoptotic/mytogenic pathways during human heart development. Int J Cardiol 96: 409-417, 2004

14. Green DR and Reed JC: Mitochondria and apoptosis. Science 281: $1309-1312,1998$.

15. Westerfield M (ed): The Zebrafish Book: A guide for the laboratory use of zebrafish Danio (Brachydanio) rerio. 4th edition. University of Oregon Press, 1993.

16. Kimmel CB, Ballard WW, Kimmel SR, Ullmann B and Schilling TF: Stages of embryonic development of the zebrafish Dev Dyn 203: 253-310, 1995.

17. $\mathrm{Xu} \mathrm{F}, \mathrm{Li} \mathrm{K}$, Tian M, et al: $\mathrm{N}-\mathrm{CoR}$ is required for patterning the anterior-posterior axis of zebrafish hindbrain by actively repressing retinoid signaling. Mech Dev 126: 771-780, 2009.

18. Nasevicius A and Ekker SC: Effective targeted gene 'knockdown' in zebrafish. Nat Genet 26: 216-220, 2000.

19. Vermes I, Haanen C, Steffens-Nakken $H$ and Reutelingsperger C: A novel assay for apoptosis. Flow cytometric detection of phosphatidylserine expression on early apoptotic cells using fluorescein labelled Annexin V. J Immunol Methods 184: 39-51, 1995.

20. Sundaresan M, Yu ZX, Ferrans VJ, Irani K and Finkel T: Requirement for generation of $\mathrm{H}_{2} \mathrm{O}_{2}$ for platelet-derived growth factor signal transduction. Science 270: 296-299, 1995.

21. Kaaman M, Sparks LM, van Harmelen V, et al: Strong association between mitochondrial DNA copy number and lipogenesis in human white adipose tissue. Diabetologia 50 2526-2533, 2007.

22. Bakkers J: Zebrafish as a model to study cardiac development and human cardiac disease. Cardiovasc Res 91: 279-288, 2011

23. Wang X, Zhou L, Jin J, et al: Knockdown of FABP3 Impairs Cardiac Development in Zebrafish through the Retinoic Acid Signaling Pathway. Int J Mol Sci 14: 13826-13841, 2013.

24. Andreyev AY, Kushnareva YE and Starkov AA: Mitochondrial metabolism of reactive oxygen species. Biochemistry (Mosc) 70 200-214, 2005

25. Akazawa $\mathrm{H}$ and Komuro I: Cardiac transcription factor Csx/Nkx2-5: Its role in cardiac development and diseases. Pharmacol Ther 107: 252-268, 2005.

26. Naito AT, Shiojima I, Akazawa H, et al: Developmental stage-specific biphasic roles of Wnt/beta-catenin signaling in cardiomyogenesis and hematopoiesis. Proc Natl Acad Sci USA 103: 19812-19817, 2006
27. Tzahor E: Wnt/beta-catenin signaling and cardiogenesis: timing does matter. Dev Cell 13: 10-13, 2007.

28. Ueno S, Weidinger $\mathrm{G}$, Osugi $\mathrm{T}$, et al: Biphasic role for Wnt/beta-catenin signaling in cardiac specification in zebrafish and embryonic stem cells. Proc Natl Acad Sci USA 104: 9685-9690, 2007.

29. Stainier DY and Fishman MC: The zebrafish as a model system to study cardiovascular development. Trends Cardiovasc Med 4: 207-212, 1994

30. Stainier DY: Zebrafish genetics and vertebrate heart formation Nat Rev Genet 2: 39-48, 2001.

31. Pelster B and Burggren WW: Disruption of hemoglobin oxygen transport does not impact oxygen-dependent physiological processes in developing embryos of zebra fish (Danio rerio). Circ Res 79: 358-362, 1996.

32. Fisher SA, Langille BL and Srivastava D: Apoptosis during cardiovascular development. Circ Res 87: 856-864, 2000.

33. McCann CJ, Glover BM, Menown IB, et al: Novel biomarkers in early diagnosis of acute myocardial infarction compared with cardiac troponin T. Eur Heart J 29: 2843-2850, 2008.

34. Lyons I, Parsons LM, Hartley L, et al: Myogenic and morphogenetic defects in the heart tubes of murine embryos lacking the homeo box gene Nkx2-5. Genes Dev 9: 1654-1666, 1995.

35. Harvey RP, Lai D, Elliott D, et al: Homeodomain factor Nkx2-5 in heart development and disease. Cold Spring Harb Symp Quant Biol 67: 107-114, 2002.

36. Biben C and Harvey RP: Homeodomain factor Nkx2-5 controls left/right asymmetric expression of bHLH gene eHand during murine heart development. Genes Dev 11: 1357-1369, 1997.

37. Eisenberg CA and Eisenberg LM: WNT11 promotes cardiac tissue formation of early mesoderm. Dev Dyn 216: 45-58, 1999.

38. Marvin MJ, Di Rocco G, Gardiner A, Bush SM and Lassar AB: Inhibition of Wnt activity induces heart formation from posterior mesoderm. Genes Dev 15: 316-327, 2001

39. Schaap FG, van der Vusse GJ and Glatz JF: Evolution of the family of intracellular lipid binding proteins in vertebrates. Mol Cell Biochem 239: 69-77, 2002.

40. Glatz JF and van der Vusse GJ: Cellular fatty acid-binding proteins: their function and physiological significance. Prog Lipid Res 35: 243-282, 1996.

41. Wang Z, Shu W, Lu MM and Morrisey EE. Wnt7b activates canonical signaling in epithelial and vascular smooth muscle cells through interactions with Fzd1, Fzd10, and LRP5. Mol Cell Biol 25: 5022-5030, 2005

42. Dohn TE and Waxman JS: Distinct phases of Wnt/ $\beta$-catenin signaling direct cardiomyocyte formation in zebrafish. Dev Biol 361: 364-376, 2012. 